\title{
Introducing a New Event in Post Herpetic Neuralgia Traditional Medicine Treatment
}

\author{
Majid Avijgan*, Seyedeh Tahoora Hajzargarbashi \\ Infectious and Tropical disease, Department of Infectious and Tropical Diseases, Department of Persian \\ Medicine, Isfahan University of Medical Sciences, Isfahan, Iran
}

*Corresponding Author: Majid Avijgan, Infectious and Tropical disease, Department of Infectious and Tropical Diseases, Department of Persian Medicine, Isfahan University of Medical Sciences, Isfahan, Iran,Email:avijgan@yahoo.com

\begin{abstract}
Post Herpetic Neuralgia (PHN) is a painful complication of herpes zoster that remain after reliving of acute phase of disease. In Traditional Chinese Medicine (TCM) PHN is due to qi and toxic pathogen stagnation, unclearing of heat and damp that leading to stagnation or obstruction of meridians. So several method of TCM is used to regulate qi and blood movement. In this review we discuss a case of PHN and treatment approach of him with different methods of TCM. We also describe a new event we observed in most treated cases that is worsening of pain by starting first cupping session of treatment but disappearing completely with continuation of acupuncture and cupping that named paradoxical phenomen (Avijgan sign).
\end{abstract}

Keywords: Post herpetic neuralgia, Traditional Chinese medicine, Acupuncture, Paradoxical phenomen.

\section{INTRODUCTION}

Varicella zoster virus (VZV) primary infection cause chicken pox and after recovery of signs and symptoms of zoster, virus can staying in neural ganglions [1]. Some triggers can reactivate the latent virus and cause dermatomal skin rash that are most appear in thoracic, trigeminal, lumbar and cervical dermatomes. The lesions don't cross the midline and depend on severity of involvement take 2-3 weeks long.

In most of severe cases that occur in cell mediated immunity impaired patients it along with Post herpetic neuralgia (PHN) [2]. PHN is defined as a neuropathic pain with different type of pain that remain 3-4months after acute phase of herpes zoster [1]. Pathophysiology of PHN is come back to neural electrolyte channels disturbances and destruction of nerves in peripheral neural system [3]. Different type of pain in PHN is included reduction of sensation, pricking pain, burning sensation and allodynia (pain without stimulator) [1], [4]. PHN in Traditional Chinese medicine (TCM) describe due to liver qi stagnation, heat stagnation and spleen disharmony [5] and has three patterns: first is damp-heat type that primary lesions occur in lower parts of body, second is wind type that lesions are in upper parts of body and third is fire or heat type (qi/blood stagnation) that are most in trunk area. Most of clinical cases of PHN are place in third type [6]. In western medicine there are several medication for PHN such as tricyclic antidepressant (nortriptyline), anti-convulsive (pregabalin or gabapentin), opioid, neurolytic blocks, local anesthesia and sometimes corticosteroid [1], [4]. In TCM there are some practical approach which discuss further [6]. This report presents a patient suffering from PHN in thoracic area for 3 and half months duration. Although several local and systemic medications were used, he had severe pain resulting to severe debility and disturbance in his life style.

The main purpose of this case report is multiple and may including following item. We will discuss about therapy of PHN with TCM approaches. With several cases we had done [6], we declare that different methods of TCM can completely cure PHN. In addition for patients who have Chronic PHN (for several years) that cause them debility it would be an effective therapeutic approach. We also present a case that despite administration of corticosteroid therapy for his persistent pain of PHN, he hasn't any response to medication but acupuncture can cure him completely. 
The method we use for this patient is acupuncture plus pricking and cupping that we confront with a new event that we named it "Avijgan sign or paradoxical phenomena". Thisis an exacerbation of pain during of treatment course. We assure both patient and physician in these cases that if they encounter with an exacerbation of pain after initiation of treatment sessions of acupuncture, don't be afraid and continue their therapeutic plan with no doubt. In the best of our knowledge, "Avijgan sign or a paradoxical phenomenon" is for the first time that is presented in medical literature.

\section{Case Presentation}

The patient is a 75 years old male who referred to our clinic suffering from persistent pain in the area of previous zoster infection. In his first visit he explain that about 105 days ago he suffered from an acute phase of varicella zoster virus infection in his left T6-T10 thoracic dermatomes from the posterior to anterior midline that presented with maculopapular and then with vesiculopustular lesions. In the course of varicella zoster presentation he used systemic antiviral (tab acyclovir $800 \mathrm{mg}$ q4h while awake for 5 times daily for 10days) and corticosteroid (tab prednisolone $50 \mathrm{mg}$ with tapering every weeks). He also used Aloe Vera Gel in ulcerative phase of lesions that after 10 days of Aloe Vera Gel, the lesions subsided but there was a persistent pain in the affected area [7]. For relieving pain he used several medications that include Amitriptyline 50mg daily, Gabapentin $300 \mathrm{mg}$ twice daily, Nonsteroidal antiinflammatory drugs (NSAIDs), local care include lidocaine ointment and capsaicin ointment and continue corticosteroids. Despite the use of these agent the patient suffer from severe pain in the lesions area. The pain cause severe debility in patient and disturbance in daily work and life mind activities so the patient had to rest or lied in bed. He indicated to not have a good night sleep during last 3.5 months. Main symptoms include severe pain in affected area, allodynia, hyperstasia exaggerated by mild touch, low appetite, weakness, fatigue, poor memory, poor concentration, mental restlessness, severe insomnia, easy to wake up, get angry easily, hypochondriac pain and depression. Based on Behavioral Rating Scale -6 (BRS-6) scoring [8] of pain, he acquired grade 6 of pain. At present physical examination, the clinical feature of lesion include hyper pigmentation compatible with dermatome of T6-
T10 from left posterior midline extended to anterior midline. He had a history of diabetes mellitus and hypothyroidism and he consumed long term corticosteroid after starting of herpes zoster. The tongue examination shows purple spots of the tongue with thin and white coat and swelling accompanied by teeth mark. The pulse examination indicates more superficial, thick, hard and forceful pulse in middle position of right pulse than left pulse but it is regular with normal rate. According to finding of pulse and tongue examination diagnosis may be qi and blood stagnation [9]. With this history and physical examination we designed a treatment plan.

Surrounding needling method of ashi points and huatijiaji point were the first choice of treatment. The acupuncture points were as follows: ashi points, Huatojiaji points at the level of T6-T10 plus two points above and below of involved area. GB34, Sj6, Sj17, liv3 plus auriculotherapy (shenmen and endocrine points).

First we started acupuncture using needles 0.25 $\mathrm{mm}$ in diameter and $40 \mathrm{~mm}$ in length (Huanqiu manufacture, China) for puncturing the mentioned points. The other method of controlling pain is surrounding method as a local technique for PHN pain. In one course, the needles were kept for 30 min once a day for 10 days consequently [6]. The standard course for this treatment is between 10 sessions, but as the patient had a complete recovery, he did not wish to continue his treatment [6].

In the first day of treatment, he had no reduction in pain. The second day was accompanied with a little reduction of pain severity. Since third day of treatment, electro acupuncture also used (G6805-2 type electric stimulator with $80 \mathrm{~Hz}$ frequency). In the fourth wet cupping method was added. In fifth days of treatment, and after cupping and bloodletting method patient suffered a severe pain and his pain exacerbated in fifth day but by continuing these methods in sixth day, the pain gradually diminish from grade4 to grade2. In the sixth day, he had complete improvement in pain sensation and also recovery of hypoesthesia and paresthesia and allodynia. In the seventh day patient had a suitable sleep state in night and after 6 months of insomnia and for the first time, he could sleep between 10pm till 4am.In the eighth day, patient could do his work in home. In the tenth day, he had a comfortable sleep and relaxes without pain. 
As described during the course at the beginning of cupping treatment we have an exacerbation of pain that with continuation of entire course of treatment plan, pain gradually decrease and patient back to his normal life without debility that we name this as "Avijgan sign or paradoxical phenomen" that is a normal part of treatment process. In the best of our knowledge, this is for the first time which this sign is presented in medical literature. This is resulted from the previous experiences in treatment of hundreds of case of PHN.

\section{DISCUSSION}

In this report we explain a patient with fire or heat type of PHN that is due to qi and blood stagnation, in this type of PHN is better to determine pain type in each point of affected area for better management [6]. Simultaneous needling in the center of painful area can relief pain [10]. Surrounding needling that in this method needle inserted at $2 \mathrm{~cm}$ intervals subcutaneously at a15 degree angle horizontally around the rash of PHN, after insertion of filiform needles, small amplitude of lifting, thrusting, twirling and rotating was done. This method can rise dispersing stasis of qi and remove obstruction of collaterals $[2,6]$.

The acupoints can feed yin and reduce fire and also can move blood to have a therapeutic effect. One of these acupoints is Jiaji. Needling of this acupoint (Jiaji) that is located in back have an analgesic effect and produce antiinflammatory mediators [6]. Electro acupuncture (EA) also can facilitate blood circulation in meridians and regulate their function and control the pain $[6,11]$. Pricking and cupping also have same pathophysiology and regulate qi and clean up heat and damp [6, 12].

In this case the day after the first cupping moving there is a worsening of pain which lead to even more complaint but reassuring the patients that it would be better with completion of treatment course and trust of patients to treatment make him prepared for accepting of pain. This kind of pain will be disappeared within one to two days and not any contraindication for further cupping. We named this type of exacerbation of pain as "Avijgan sign" which is one of the signs of response of patients to treatment. In the best knowledge this is the first time that this phenomen is reported [13] and needs further evaluation by further clinical cases.

\section{Conclusion}

The exacerbation of PHN Pain after starting cupping or "Avijgan sign or Paradox phenomen" is not contraindication for continue treatment of PHN. It is better to assure the patients to accept this pain for one or two days which is a good prognosis for pain outcome.

\section{REFERENCES}

[1] J. I. Cohen, "Herpes Zoster," N. Engl. J. Med., vol. 369, no. 3, pp. 255-263, Jul. 2013.

[2] X. Fang, "Clinical observation of acupuncture therapy for postherpetic neuralgia," $J$. Acupunct. Tuina Sci., vol. 9, no. 2, p. 113, Apr. 2011.

[3] E. M. Garry et al., "Varicella zoster virus induces neuropathic changes in rat dorsal root ganglia and behavioral reflex sensitisation that is attenuated by gabapentin or sodium channel blocking drugs," Pain, vol. 118, no. 1, pp. 97111, Nov. 2005.

[4] R. W. Johnson and A. S. C. Rice, "Postherpetic Neuralgia," N. Engl. J. Med., vol. 371, no. 16, pp. 1526-1533, 2014.

[5] M. Avijgan and M. Avijgan, "Can the primo vascular system (Bong Han Duct System) be a basic concept for Qi production?," Int. J. Integr. Med., vol. 1, no. 20, pp. 1-10, 2013.

[6] M. Avijgan, S. T. Hajzargarbashi, A. Kamran, and M. Avijgan, "Postherpetic Neuralgia: Practical Experiences Return to Traditional Chinese Medicine," J. Acupunct. Meridian Stud., vol. 10, no. 3, pp. 157-164.

[7] M. Avijgan, "Aloe Vera Gel as an Effective and Cheap Option for Treatment in Chronic Bed Sores," Gums-Med, vol. 13, no. 50, pp. 4551, Jul. 2004.

[8] M. P. Jensen, P. Karoly, and S. Braver, "The measurement of clinical pain intensity: a comparison of six methods," Pain, vol. 27, no. 1, pp. 117-126, 1986.

[9] M. Avijgan, K. Shirani, and M. Meidani, "Diagnosis of pulmonary thromboemboli by tongue examination: an example for integration of Traditional Chinese Medicine and modern medicine," J. Exp. Integr. Med., vol. 2, no. 2, pp. 185-188, 2012.

[10] G. T. Lewith, J. Field, and D. Machin, "Acupuncture compared with placebo in postherpetic pain," Pain, vol. 17, no. 4, pp. 361368, 1983. 
[11] S. Yuan-zheng and M. Jin-yun, "Treatment of 28 Cases of Post-herpetic Neuralgia by Local Encirled Needling Method," J. Acupunct. Tuina Sci., vol. 4, no. 4, pp. 250-251, Aug. 2006.

[12] L. Yong-qing, "Treatment of 17 cases of herpes zoster by pricking and cupping method," $J$. Acupunct. Tuina Sci., vol. 1, no. 5, pp. 46-46, Oct. 2003.
[13] Avijgan M. Management of Post Herpetic Neuralgia: Brief report of Practical Actions. Int J Complement Alt Med. 11(3):00351. (2018). DOI: 10.15406/ijcam.2018.11.00351

Citation: Majid Avijgan, Introducing a New Event in Post Herpetic Neuralgia Traditional Medicine Treatment.ARC Journal of Dermatology. 2018; 3(2):8-11. doi:dx.doi.org/10.20431/2456-0022.0302003.

Copyright: (c) 2018 Authors. This is an open-access article distributed under the terms of the Creative Commons Attribution License, which permits unrestricted use, distribution, and reproduction in any medium, provided the original author and source are credited. 